\title{
Protótipo de Apoio ao Ensino e Aprendizagem de Endometriose por meio de Dispositivos Móveis
}

\author{
Alexandre Freitas Duarte ${ }^{1}$, Nemésio Freitas Duarte Filho², Ellen Francine \\ Barbosa $^{2}$, Norben Paulus de Oliveira $\operatorname{Costa}^{3}$ \\ ${ }^{1}$ Faculdade de Filosofia Ciências e Letras (FFCLRP/USP) - Universidade de São Paulo \\ Caixa Postal 14.015-000 - Ribeirão Preto - SP - Brasil \\ ${ }^{2}$ Instituto de Ciências Matemáticas e de Computação (ICMC/USP) - Universidade de São Paulo \\ Caixa Postal 13.566-590 - São Carlos - SP - Brasil \\ ${ }^{3}$ União das Instituições Educacionais do Estado de São Paulo (UNIESP) \\ Caixa Postal 14.010-060 - Ribeirão Preto - SP - Brasil \\ alexfd7@gmail.com, \{nemesio, francine\}@icmc.usp.br, shadow_suporte@hotmail.com
}

\begin{abstract}
Mobile learning systems emerge as an important alternative to enhance teaching and learning virtual, incorporating greater motivation, mobility and collaboration between learners and tutors. In this context, mobile learning has been used in medicine, flexing the dissemination of knowledge designed for students, professionals and even patients. This dissemination of knowledge enables support the teaching, along with conducting campaigns on prevention of diseases and epidemics. Therefore, this work presents the development of a prototype mobile learning with a focus on supporting teaching and learning about endometriosis.
\end{abstract}

Resumo. Sistemas de aprendizagem móvel surgem como uma importante alternativa de potencializar o ensino e a aprendizagem virtual, incorporando maior motivação, colaboração e mobilidade entre os aprendizes e tutores. Neste contexto, a aprendizagem móvel vem sendo utilizado na área da medicina, flexibilizando a disseminação de conhecimento direcionado para estudantes, profissionais da área e até mesmo pacientes. Esta disseminação de conhecimento permite apoiar o ensino, juntamente com a realização de campanhas sobre prevenção de doenças e epidemias. Deste modo, este trabalho apresenta o desenvolvimento de um protótipo de aprendizagem móvel com foco no apoio ao ensino e aprendizagem sobre endometriose.

\section{Introdução}

Os sistemas e ambientes de aprendizagem têm apresentando uma crescente importância nos últimos anos, tornando-se fundamental para o ensino e treinamento, sendo relevantes em ambientes acadêmicos, organizacionais e até mesmo dentro da área de ciências médica. Partindo deste raciocínio muitas organizações e instituições estão cada vez mais utilizando sistemas de apoio ao ensino, proporcionando maior treinamento e aperfeiçoamento de aprendizes, sejam eles pesquisadores, profissionais da área da saúde ou pacientes. 
Com o avanço tecnológico e o grande desenvolvimento de tecnologias móveis e sem fio, é possível a percepção de uma crescente necessidade por mobilidade de pessoas, objetos e informação [Kristoffersen et al. 2000]. Juntamente com o advento e o desenvolvimento da computação ubíqua, vêm surgindo um novo modelo de aprendizagem, a aprendizagem móvel (m-learning) [Caudill 2007]. Este novo modelo proporciona uma maior interação e colaboração entre aprendizes, tutores, e outros profissionais, como por exemplo, médicos e seus pacientes.

No Brasil, a área médica devido a sua complexidade de conhecimento e informações, é necessário e útil a utilização de sistemas computacionais, com o objetivo de auxiliar as atividades dos profissionais de saúde, como por exemplo: suporte a tomada de decisões, treinamento, acompanhamentos clínicos e gestão em geral. Os sistemas computacionais com foco no apoio ao ensino e treinamento também ajudam a flexibilizar a disseminação de conhecimento para os pacientes, ajudando na prevenção e esclarecimento sobre doenças.

Como exemplo da utilização da aprendizagem móvel na medicina, podemos destacar campanhas de prevenção no tratamento de alguma doença, até então raras ou cujo objetivo é orientar a população sobre métodos preventivos e sintomas. O modelo de aprendizagem móvel possibilita aos usuários contribuir, participar e acessar o ambiente de ensino através de dispositivos móveis, tais como celulares, i-pods, laptops, $t v$, entre outros, a qualquer momento e em qualquer lugar [Svetlana et al. 2009].

$\mathrm{Na}$ área médica, a endometriose é uma doença que acomete cerca de $10 \%$ das mulheres em idade reprodutiva. Os sintomas mais comuns são dores pélvicas e infertilidade [Podgaec et al. 2004]. A causa da endometriose é desconhecida e complexa, e existem diversas teorias e tipos de diagnósticos [Lobo 2007]. Pela gravidade que a doença pode representar, já existem campanhas realizadas no Brasil com o objetivo de esclarecimento para pessoas, com intuito de disseminar um melhor conhecimento e possibilitar um diagnóstico precoce. Tais campanhas são importantes, vendo que no Brasil a endometriose tem sido diagnosticada nas mulheres com atraso, tornando este fato um problema de saúde pública.

Dentro deste contexto, este artigo apresenta uma proposta de desenvolvimento de um protótipo de aprendizagem móvel com foco no ensino e treinamento de endometriose, proporcionando uma melhora na disseminação do conhecimento desta doença. O protótipo foi avaliado com a ajuda de especialistas (médicos) e usuários comuns, sendo identificados tanto aspectos positivos como negativos em sua utilização.

O artigo encontra-se organizado como se segue. A Seção 2 apresenta a base teórica sobre aprendizagem móvel e endometriose. A Seção 3 traz a visão geral do protótipo, descrevendo o seu processo de desenvolvimento e principais funcionalidades. A Seção 4 apresenta os resultados detalhados, obtidos após as avaliações do sistema. Por fim, a Seção 5 apresenta a conclusão e trabalhos futuros. 


\section{Referencial Teórico}

A seguir alguns aspectos sobre aprendizagem móvel e endometriose são abordados.

\subsection{Aprendizagem Móvel (m-learning)}

Com o advento e evolução das tecnologias de informação, juntamente com a computação ubíqua, é possível observar uma nova modalidade de ensino, baseada na computação ubíqua ou computação móvel, denominada aprendizagem móvel ou mobile learning (m-learning) [Zare 2011]. Conceitualmente o m-learning pode ser definido como qualquer tipo de ensino ou treinamento que ocorre quando um aprendiz não está em um lugar predeterminado ou fixo, ou quando o indivíduo tira partido de oportunidades de aprendizagem disponibilizadas pelos dispositivos móveis [Moura 2009].

Por se tratar de um paradigma novo e emergente, há diversas tentativas para definir m-learning. Rachid and Ishitani [2012] definem m-learning como qualquer tipo de ensino ou aprendizagem que ocorre quando o aluno não está em algum lugar fixo, ou quando o indivíduo aproveita-se de oportunidades de aprendizagem disponibilizadas pelas tecnologias móveis, associando assim os conceitos tecnológicos e de mobilidade. Ozdamli and Cavus [2011] abordam m-learning como uma atividade que permite aos indivíduos serem mais produtivos quando consomem, criam ou interagem com as informações, mediados por dispositivos digitais móveis e portáteis.

Este novo paradigma de ensino além de facilitar a aprendizagem de seus usuários, podem também ser utilizado para uma aprendizagem no trabalho. Assim os usuários trabalhadores podem passar mais tempo onde necessitam estar, e também dedicando seu tempo à atualização de seu conhecimento e uma possível formação continuada [Koschembahr 2000]. Porém, por ser um modelo novo e emergente, os sistemas de m-learning podem apresentar algumas limitações em utilização, como por exemplo: poder de processamento reduzido; tela de tamanho; energia limitada (dependente de baterias); comunicação com taxas de transmissão, geralmente, menores do que as das redes fixas; aspectos de usabilidade; entre outras [Pachler 2007].

Independentemente da definição adotada e eventuais limitações de uso, a utilização de sistemas de aprendizagem por meio de dispositivos móveis traz benefícios que vão além de acessibilidade, comodidade e comunicação [Schepman et al. 2012]. Por exemplo, com dispositivos móveis os aprendizes podem utilizar aplicativos (processamento de texto, fotos), ambientes específicos de aprendizagem, acesso a Web, ferramentas de colaboração, redes sociais, entre outros.

Por meio dos benefícios proporcionados pela aprendizagem móvel, já é possível observar e identificar iniciativas na literatura da aprendizagem móvel na área da medicina [Paiva et al. 2013; Galvão et al. 2012], flexibilizando o ensino e o treinamento de aprendizes e pacientes. A aprendizagem móvel aplicada diretamente na medicina também proporciona a melhora da disseminação do conhecimento, em relação à prevenção de doenças e campanhas temáticas. 


\subsection{Endometriose}

Endometriose é uma condição na qual o tecido que age como a mucosa que reveste a parede interna do útero (endométrio) cresce em outras regiões do corpo, essa formação de tecido normalmente ocorre na região pélvica, fora do útero, nos ovários, no intestino, no reto, na bexiga e na delicada membrana que reveste a pélvis. Quando essas células (chamadas de células endometriais) crescem fora do útero, surge a endometriose. Ao contrário das células normalmente encontradas dentro do útero que são liberadas durante a menstruação, as células fora do útero permanecem no lugar, às vezes, sangram um pouco, mas se curam e são estimuladas novamente durante o ciclo seguinte [Lobo 2007].

Segundo Podgaec et al. [2004] a endometriose afeta cerca de $10 \%$ das mulheres em idade reprodutiva e os sintomas mais comuns são dores pélvicas e infertilidade. Normalmente, portadoras desta doença sofrem uma diminuição substancialmente em suas qualidades de vida, uma vez que a dor pélvica é frequente e muitas vezes incapacitante até para realizar as tarefas mais simples, acarretando num significativo desgaste físico. Esta enfermidade é caracterizada como uma doença complexa e enigmática do corpo humano. Existem diversas teorias e tipos de diagnósticos, sendo merecida de uma melhor atenção parte dos médicos e ginecologistas, cujo objetivo é cuidar da saúde e oferecer qualidade de vida às mulheres [Lobo 2007].

Segundo a Sociedade Brasileira de endometriose (SBE) ${ }^{1}$, a postergação da maternidade pode ser um dos fatores do desencadeamento da doença, porém, a causa da endometriose ainda é totalmente desconhecida e complexa, e existem diversas teorias e tipos de diagnósticos. Pela gravidade que a doença pode representar, já existem campanhas realizadas no Brasil com o objetivo de esclarecimento para pessoas e de reciclagem para médicos, com intuito de disseminar um melhor conhecimento e possibilitar um diagnóstico precoce, já que no Brasil a endometriose tem sido diagnosticada nas mulheres com atraso, tornando este fato um problema de saúde pública.

Neste contexto, algumas iniciativas computacionais vêm sendo realizadas proporcionando sistemas de apoio à decisão e sistemas de gestão referentes ao tema de endometriose. Não sendo encontrados sistemas e ambientes de apoio ao ensino e aprendizagem, possibilitando a sua utilização por partes de aprendizes (promovendo a capacitação dos futuros profissionais) e pacientes (promovendo a disseminação de conhecimento e prevenção).

\section{Visão Geral do Protótipo}

O protótipo funcional desenvolvido neste trabalho é um sistema de aprendizagem móvel, cujo objetivo é prover uma formação continuada aos aprendizes que buscam atualizar seus conhecimentos sobre o tema de endometriose e também potencializar uma melhora no esclarecimento de pessoas, dando auxílio nas campanhas de prevenção e tratamento da doença. Os usuários em sua maioria são graduandos (jovens entre 18 e 30 anos), sendo também de usufruto à profissionais de saúde e pacientes, todos estes

\footnotetext{
${ }^{1}$ http://www.Endometriose.org.br/site_abend/site/home.asp - Sociedade Brasileira de Endometriose
} 
utilizam o sistema para consultar informações e atualizar seus conceitos sobre temas específicos à endometriose.

\subsection{Processo de Desenvolvimento}

O modelo de processo de desenvolvimento foi definido em quatro etapas:

1.Concepção e Definição do Escopo: uma das atividades iniciais com muita importância de uma trabalho científico é a definição da problemática. A definição surgiu a partir das necessidades e carências encontradas na comunidade e no meio científico, como na área médica, especificamente com o tema endometriose, no qual apresenta carência e precariedade em relação a um sistema de apoio ao ensino, melhorando a formação dos profissionais de saúde e disseminação de conhecimento perante os pacientes.

2.Projeto de Interface do Protótipo: a construção de interfaces é uma peça fundamental para um sistema computacional, sendo desejável que ela seja estruturada e adaptada para sistemas móveis, flexibilizando a utilização das práticas educacionais. Para esta etapa o escopo do design foi definido por meio das seguintes atividades: (1) definição dos requisitos a serem implementados; (2) definição da infraestrutura de hardware e software para implementação do protótipo; e (3) definição das opções/contrapartidas a serem consideradas como limitações do protótipo. Após esta atividade, interfaces foram ilustradas com o objetivo de identificar pontos positivos e negativos em relação à usabilidade do sistema. Três ilustrações foram confeccionadas utilizando sketches e storyboard. Para cada interface desenhada, foi descrito um cenário (na forma de texto), descrevendo como os usuários utilizariam o sistema.

3.Definição e Desenvolvimento: a construção do protótipo foi realizado na plataforma Android, possuindo uma distribuição gratuita. Os requisitos funcionais necessários a serem incluídos no protótipo foram levantados a partir de consultas em trabalhos relacionados à aprendizagem móvel e demonstrações de sistemas prontos com foco na área médica. Para a confecção do material didático, foi efetuado um levantamento de conteúdo e informações pertinentes ao tema endometriose através de consultas à literatura e entrevistas com profissionais especialistas da área.

4.Avaliação: para evidenciar os resultados finais, foram realizados testes de usabilidade (técnica de ThinkAloud) com especialistas da área e usuários comuns, possibilitando a identificação de limitações e possíveis melhorias no protótipo. Nas próximas seções serão apresentados com mais detalhes o processo de avaliação do protótipo, juntamente com a utilização da técnica de ThinkAloud.

\subsection{Principais Características e Funcionalidades}

As principais características e funcionalidades do protótipo são apresentadas a seguir.

Interface de Login: permite um controle através da identificação do usuário, por meio da inserção de um usuário e senha.

Interface de Cadastro: usuário sem permissão ao sistema pode realizar o seu cadastro, preenchendo os itens básicos: nome completo, nome do usuário, senha, confirmação de senha, e-mail e confirmação de email. 
Interface Central: após realizar o acesso, é apresentado ao usuário a área central do ambiente (Figura 1). Nesta interface são listados todos os cursos disponíveis pelo sistema, nas quais os usuários poderão usufruir dos mesmos de acordo com seus interesses. Informações mais detalhadas sobre cada curso são apresentadas, como por exemplo: avaliações feitas pelos usuários (representados por estrelas) e quantidade de acessos obtidos, sendo localizadas respectivamente ao lado direito da tela. Ressalta-se que o protótipo foi elaborado possibilitando a inclusão de diferentes cursos, cujo objetivo futuro é a disponibilização de um repositório de cursos específicos para a área médica. Porém, neste trabalho o foco será somente em relação ao tema endometriose.

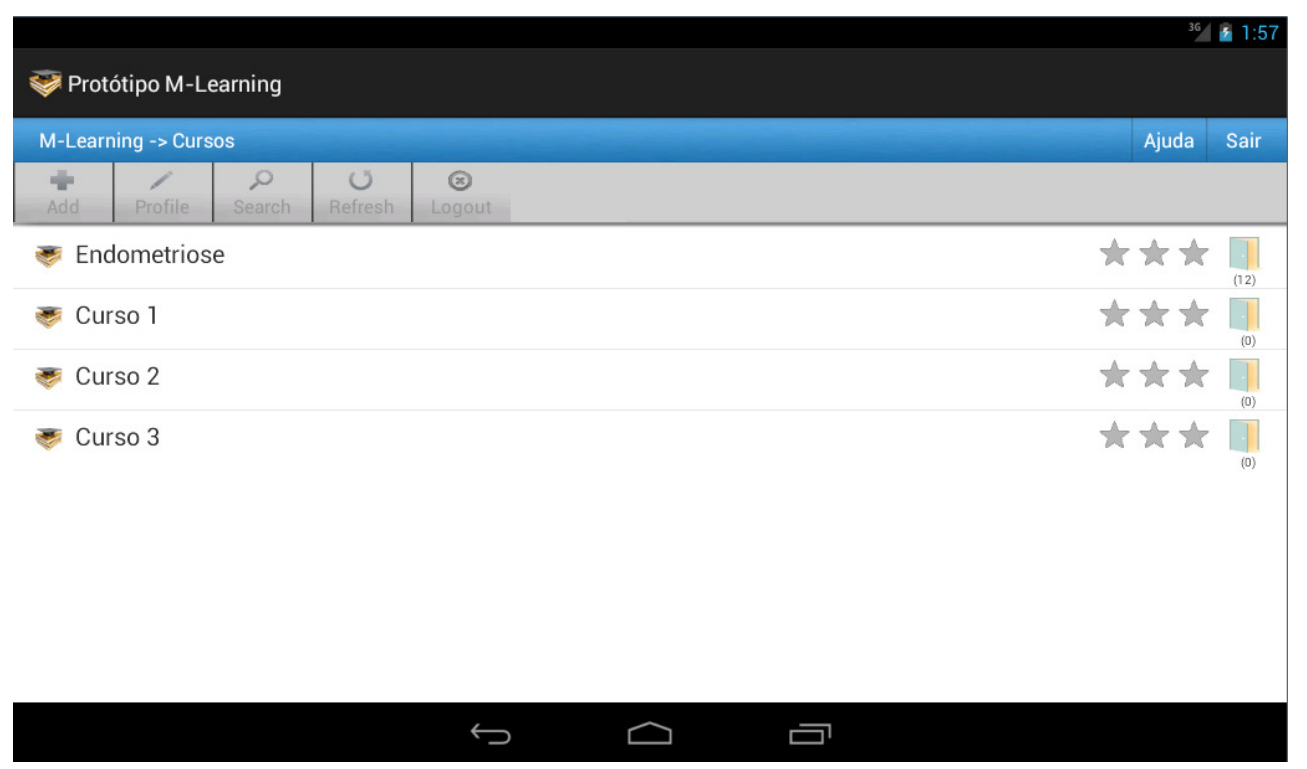

Figura 1 : Tela Central

Ainda em relação à interface principal, encontra-se uma importante região representada por uma barra de botões, que provê funcionalidades específicas ao usuário, sendo elas: (1) Botão $A d d$, realiza a inclusão de novos cursos por parte de administradores e usuários com permissões específicas; (2) Botão Profile, permite a visualização e edição dos dados dos usuários; (3) Botão Search, permite a realização de pesquisas, facilitando a busca ao longo de todo os cursos disponíveis no sistema; (4) Botão Refresh, útil pois atualiza as informações na tela quando acionado, vendo que as informações podem periodicamente ser criadas, alteradas e apagadas por outros usuários e administradores; e (5) Botão Logout, encerrando a sessão do usuário.

Interface de Aprendizagem: Em relação ao material didático, o conteúdo foi dividido em tópicos, e estes estão disponibilizados de uma forma sequencial de acordo com o estudo realizado anteriormente na fase de definição e desenvolvimento. Este estruturação é importante, pois permite uma navegação de conhecimento de maneira progressiva, possibilitando a aprendizagem por níveis básicos, intermediários e avançados.

Mensagens de Aviso: o protótipo também possui mecanismos que flexibilizam a comunicação com os usuários em decorrência de erros e avisos. Caso seja necessário, mensagens pré-definidas de avisos são lançadas para os usuários, avisando-os de ações que contrariam as políticas de acesso, cadastro e funcionais. 
Conteúdo Educacional: aplicações em dispositivos móveis devem levar em consideração a limitação visual, proporcionando uma redução do campo visual na tela do dispositivo, que em comparação a uma estação de trabalho ou notebook são muito maiores.

Neste contexto, o protótipo desenvolvido, exige um grande cuidado na apresentação das informações/conteúdo para que não haja uma sobrecarga de textos na tela. É importante evitar que informações desnecessárias sejam disponibilizadas para os usuários. Para isso, caso o usuário queira adquirir um pouco mais de informação, na interface de aprendizagem os parágrafos foram criados com a opção de serem expansíveis, lançando novas informações para o usuário de acordo com o seu interesse e ao longo de sua leitura (Figura 2 - a;b).

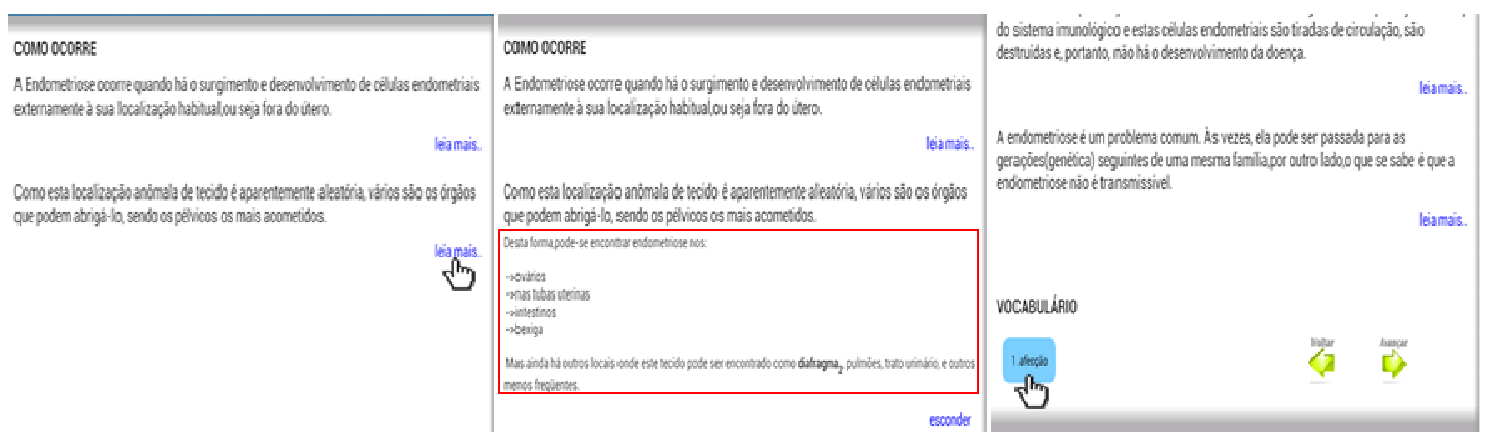

(a) Parágrafo não expandido

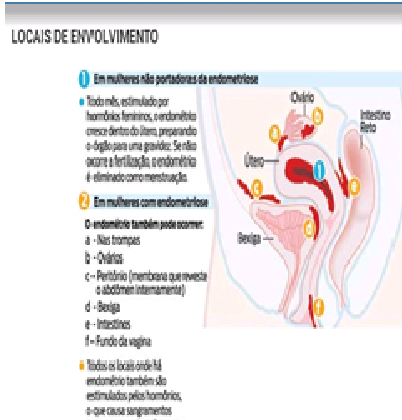

(d) Imagem (b) Parágrafo expandido

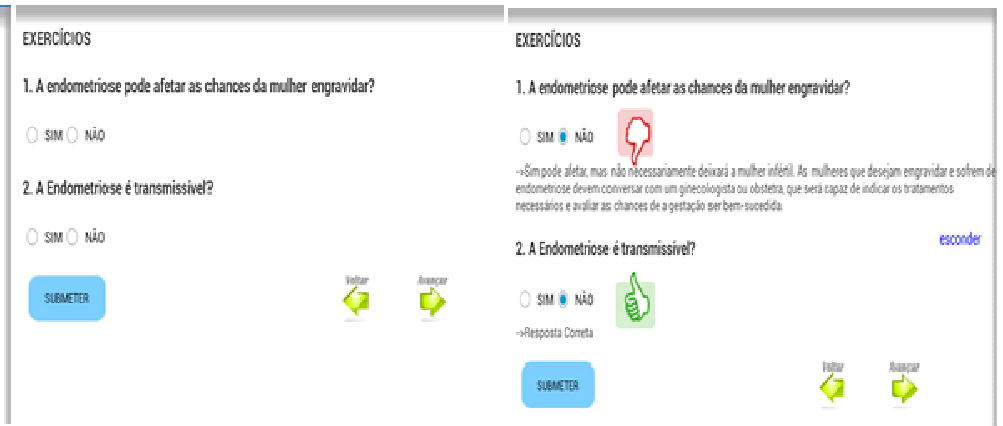

(e) Exercícios (c) Vocabulário

(f) Avaliação dos Exercícios

Figura 2 : O protótipo m-learning

Em busca de um alto nível educativo por parte do conteúdo didático, uma funcionalidade de vocabulário também foi implementada, onde palavras/terminologias relacionadas ao tema endometriose são disponibilizadas com suas definições ao final de cada página de conteúdo (Figura 2 - c). Igualmente ao vocabulário, outros recursos como imagens e vídeos foram utilizados para que torne a aprendizagem mais atrativa e flexiva (Figura 2 - d).

Com o objetivo de proporcionar maior interação do usuário com o ambiente de aprendizagem, exercícios/teste foram implementados e disponibilizados ao final de todo conteúdo (Figura 2 - e;f). A cada tópico o usuário vai adquirindo informações sobre o conteúdo e no fim ele pode testar o seu conhecimento por meio de exercícios e testes didáticos, permitindo assim, uma auto-avaliação.

Tratamento de Usabilidade: Segundo Nielsen [1993], a usabilidade é um conjunto de propriedades importantes para sistemas de ensino e aprendizagem, 
garantindo: maior eficiência, capacidade de memorização, baixo índice de erros, satisfação e prazer ao uso, entre outros. No protótipo em questão foram consideradas algumas características de usabilidade, visando maior flexibilidade e percepção frente aos usuários em relação a um sistema móvel educacional, sendo elas:

- Navegação: facilidade na utilização do aplicativo. A interface deve permitir que o usuário alcance e realize suas atividades com eficiência;

- Validação de dados: validações e filtragem de parâmetros de entrada pelo usuário são realizados para garantir consistência e integridade dos dados;

- Número de toques (touchs): houve uma preocupação em minimizar o número de touchs efetuados pelos usuários na realização das atividades. O ideal é que o usuário alcance seus objetivos com um mínimo esforço e máximo resultado; e

- Satisfação: algumas premissas foram adotadas, tendo como objetivo a busca de um alto nível de satisfação por parte dos usuários em relação à utilização de cores e disposição dos elementos na tela. Houve a preferência pela a utilização de espaçamentos em branco (diminuindo a poluição visual), adoção de fontes e textos adequados e inserção de botões grandes, vendo que os usuários utilizarão mecanismos táteis em um dispositivo móvel.

\section{Avaliação}

Esta seção destaca a avaliação do protótipo. Os autores do trabalho utilizaram a técnica ThinkAloud com a ajuda de especialistas e usuários comuns, evidenciando pontos positivos e negativos em relação a utilização do protótipo.

\subsection{ThinkAloud}

Este teste de usabilidade consiste em estimular os participantes a verbalizarem durante o uso/realização de uma tarefa no protótipo, permitindo que o observador investigue possíveis dificuldades ou problemas que o protótipo possa apresentar.

1. Deslocamento entre tópicos do curso fazendo uso dos botões Avançar e Voltar.

2. Assistir o vídeo disponibilizado no conteúdo;

3. Realizar os exercícios disponibilizados;

4. Identificar uma palavra vinculada ao vocabulário, e acessar sua definição; e

5. Realizar a navegação completa por um tópico de conteúdo.

Este teste foi realizado utilizando um dispositivo Tablet Android 4.1.2, de definição 1024 X 600, e um aplicativo nativo de gravação de voz, que foi ativado durante a realização das tarefas pelo participante. Os testes foram executados individualmente com a participação de dois especialistas e três usuários comuns, isto levou a identificação de alguns problemas/limitações no protótipo, sendo eles:

- Ausência de funcionalidade de flip page (ou page view), permitindo ao usuário deslizar o dedo horizontalmente na tela, podendo de uma forma mais eficiente, navegar entre as páginas do conteúdo do curso. Isto ausenta ao aprendiz a 
necessidade de localizar e utilizar a lista de tópicos e/ou as setas de Avançar e Voltar para o seu deslocamento entre as páginas e conteúdo;

- Ausência de funcionalidade de pinch zoom, esta função quando implementada, proporciona ao aprendiz uma melhor visualização das imagens e textos que foram disponibilizadas ao longo conteúdo, possibilitando o seu zoom por meio do toque conjunto de dois dedos à tela sensível, aumentando e diminuindo o zoom respectivamente; e

- Ausência do número de páginas, proporcionando uma noção por parte do aprendiz de quantas páginas faltam para o término do curso.

\section{Conclusão e Trabalho Futuros}

No contexto da aprendizagem móvel em relação à aplicação médica, verifica-se uma carência e precariedade de ferramentas simples e flexíveis, que proporcionem uma aprendizagem adequada por meio de dispositivos móveis e que, ao mesmo tempo, não seja impactada pelas limitações desses dispositivos.

Devido a esta carência, este trabalho teve como objetivo o desenvolvimento e implementação de um protótipo de aprendizagem móvel, cujo objetivo é prover uma formação continuada aos aprendizes que buscam atualizar seus conhecimentos sobre o tema de endometriose e também potencializar uma melhora no esclarecimento de pessoas, dando auxílio nas campanhas de prevenção e tratamento da doença.

Os usuários do protótipo em sua maioria são graduandos (jovens entre 18 e 30 anos), sendo também de usufruto a profissionais de saúde e pacientes, todos estes utilizam o sistema para consultar informações e atualizar seus conceitos sobre temas específicos à endometriose.

Para o desenvolvimento do protótipo, algumas etapas foram consideradas, sendo elas: concepção e definição do escopo, juntamente com fundamentações teóricas e ajuda de especialistas; projeto de interface do protótipo, definindo uma interface correta e flexível ao contexto de sua utilização; definição e desenvolvimento; e ao final a avaliação, utilizando o teste ThinkAloud.

O protótipo foi implementado sendo capaz de controlar o acesso dos usuários, listar e gerenciar de uma forma organizada os cursos disponíveis, disponibilizar uma área amigável com ferramentas direcionadas à aprendizagem e prover um conteúdo didático adequado. Ao longo do desenvolvimento foram consideradas características de usabilidade a serem incluídas no protótipo, visando maior flexibilidade, motivação, adaptação e percepção por parte dos usuários.

Ao longo da avaliação do protótipo, através da aplicação da técnica de ThinkAloud com especialistas e usuários comuns, foram observados pontos positivos e negativos em relação a utilização do protótipo. Os pontos limitantes identificados e analisados ao longo das avaliações foram: (1) ausência de funcionalidades de flip page (page view); (2) ausência de funcionalidades de pinch zoom; e (3) ausência de paginação.

Como trabalhos futuros, pretende-se modificar e evoluir o protótipo possibilitando a colaboração e contribuição entre os aprendizes e tutores através da Web. 
Para isso, um fórum já vem sendo desenvolvido, incorporando dentro do protótipo a colaboração entre os seus usuários. Em segundo plano, será realizado um experimento do protótipo juntamente com estudantes de medicina e pacientes relacionados com endometriose, possibilitando coletar informações sobre a sua utilização em um ambiente real.

\section{Referências}

Caudill, J. G. (2007). growth of m-Learning and the growth of mobile computing. International Review of Research in Open and Distance Learning, v. 8.

Galvão, E. C. F., Püschel, V. A. A. (2012). Aplicativo multimídia em plataforma móvel para o ensino da mensuração da pressão venosa central. Rev. Esc. Enfermagem da USP.; 46 (Esp):10715.

Koschembahr, C. (2005). Mobile Learning: the next evolution. Chief Learning Officer, February.

Kristoffersen, S., Ljungberg, F. (2000). Mobility: from stationary to mobile work. In: BRAA, K.; SORENSEN, C.; DAHLBOM, B. (eds.) Planet Internet. Studentlitteratur, Lund, Sweden.

Lobo, R. (2007). Endometriosis: etiology, pathology, diagnosis, management. Katz VL, Lentz GM, Lobo RA, Gershenson DM. Comprehensive Gynecology. 5th ed. Philadelphia, Pa: Mosby Elsevier; chap. 19.

Moura, A. (2009). Geração Móvel: um ambiente de aprendizagem suportado por tecnologias móveis para a Geração Polegar. In P. Dias, A. J. Osório (Org.) Actas da VI Conferência Internacional de TIC na Educação Challenges 2009 / Desafios 2009. Braga: Universidade do Minho, p. 50-78.

Nielsen, J. (1993). Usability Engineering. 1 ed. Boston: Academic Press, 1 vol.

Ozdamli, F., Cavus, N. (2011). Basic elements and characteristics of mobile learning. World Conference on Educational Technology Researches, Volume 28, p 937-94.

Pachler, N. (2007). Mobile learning: towards a research agenda. Londres. Institute of Education, University of London.

Paiva, L. R. M. (2013). Aplicação para dispositivos móveis utilizando tecnologias interativas: a Realidade Virtual e Aumentada aplicada ao estudo da anatomia humana. X Encontro Anual de Computação - EnAComp-IFTM.

Podgaec, S., Abrão, M. S. (2004). Endometriose: aspectos atuais do diagnóstico e tratamento. http://www.moreirajr.com.br/revistas.asp?id_materia=2567\&fase=imprime.

Rachid, L. C., Ishitani, L. (2012). m-tutorial: ferramenta de autoria para desenvolvimento de tutoriais voltados para o m-learning. In: Revista Brasileira de Informática na Educação (RBIE), v.20, n.1.

Schepman, A., Rodway, P., Beattie, C., Lambert, J. (2012). An observational study of undergraduate students adoption of (mobile) note-taking software. Computers in Human Behavior, 28(2), 308317.

Svetlana, K., Yoon, Y. (2009). Adaptation e-learning contents in mobile environment. In: ICIS '09: Proceedings of the 2nd International Conference on Interaction Sciences. New York, NY, USA: ACM, p. 474-479. ISBN 978-1-60558-710-3.

Zare, S. (2011). Personalization in Mobile Learning for People with Special Needs. UAHCI'11 Proceedings of the 6th international conference on Universal access in human-computer interaction: applications and services - Volume Part IV, p 662-669. 